\title{
MEDICIONES PÚBLICAS REALIZADAS \\ POR ACTORES PRIVADOS: EL CASO DE \\ LOS RANKINGS MBA
}

Los MBA (Master in Business Administration) son programas que han tenido un gran auge a partir de los 90 en América Latina, y mitad de los 80 en Estados Unidos y Europa. Si bien tienen una data de origen más antigua ${ }^{1}$, ha sido en las últimas dos décadas que se han consolidado mundialmente como los programas de posgrado de mayor demanda.

En un comienzo los MBA estuvieron asociados a las áreas de administración, y cualquiera que deseara tener éxito en el mundo de los negocios necesitaba estudiar uno de estos programas. De esta manera, el concepto se fue adaptando y las escuelas de negocios entendieron que en el mercado existía la necesidad, de parte de distintas profesiones, de conocer las claves de este mundo. Así, surgen los Executive MBA con un formato más flexible y accesible, pero también más cuestionado desde el punto de vista de los programas tradicionales.

Esta explosión de programas MBA adaptados a las necesidades de cada persona ha significado que hoy exista una gran oferta, que muchas veces supera la demanda, generándose una gran asimetría en la información entregada al público, lo cual ha provocado ciertos grados de confusión.

El presente artículo tiene por objeto describir las herramientas que el mercado de MBA ha desarrollado para autorregularse y los desafíos que aún se le presentan en un medio como el chileno que está en etapa de formación.

1 El primer programa de MBA data de principios de 1900, sin embargo, el formato, tal como lo conocemos hoy, surge a partir de los 60 . 


\section{LOS RANKINGS}

Actualmente, es bastante común encontrarse con propaganda de programas de MBA donde se destacan a sí mismos como "los únicos en..." o "los $\mathrm{N}^{\mathrm{o}} 1 \mathrm{en} . .$. ". Sin embargo, a pesar de que la publicidad es una herramienta esencial en el desarrollo de cualquier mercado competitivo, esto no ha sido suficiente para que el público comprenda las diferencias de fondo entre estos programas, ni tampoco para regular su mercado.

Ante la necesidad de conocer mejor la oferta surgieron los llamados rankings de MBA, impulsados por las más importantes revistas y periódicos del mundo anglosajón dedicados a los negocios, tales como Wall Street Journal, Business Week, Finantial Times y The Economist. Éstos tienen por objeto evaluar los programas desde distintos ámbitos, como son la calidad académica, la percepción de los ejecutivos, reclutadores y ex alumnos, el retorno de la inversión, la red de contactos, y algunas otras dimensiones menos relevantes como la infraestructura, de manera que los postulantes puedan entender con mayor detalle quién es quién en este mercado.

Sin embargo, a pesar de la no despreciable cantidad de rankings de esta naturaleza que circulan por el mundo, estos no son comparables entre sí. Cada uno utiliza una metodología diferente y no necesariamente incluyen a todos los actores del mercado, ya que muchos aún se resisten a participar en este tipo de estudios que exponen a sus programas a no obtener los resultados que las escuelas esperarían.

La gran crítica a estos rankings ha sido que evalúan, como cualquier estudio de opinión, un sólo momento y no la trayectoria de los programas, lo cual lleva a que la variación de año a año pueda ser muy grande 2 . Otra fuente de crítica es que, quienes hacen estos estudios, las revistas y/o periódicos, también tendrían un fin económico: vender más ediciones y publicidad.

Sin embargo, y a pesar de estos defectos y críticas, siguen siendo eficientes a la hora de mostrar aquella información que ni las escuelas,

2 Tal como AMBA (Associations of MBAs) señala en su sitio Web: "existen muchas escuelas de buena calidad que fallan a la hora de ser evaluadas por los rankings y eso no significa que sean inferiores a "las grandes marcas". [Sitio en Internet] Disponible en http:// www.mbaworld.com/index.php? content=rankings\&mm $=13 \& s m=11$ 
ni las acreditaciones exhiben como por ejemplo, la evolución de los salarios, la opinión de las empresas, y la de los graduados. En el caso de un mercado como el nuestro, en que no existe acreditación local, son la única herramienta, perfectible aún, que muestra la situación comparada de los distintos programas, pudiendo acceder en un solo documento a información objetiva y generada por un ente independiente de los intereses de cada escuela de negocios.

Debido a la gran heterogeneidad de programas ofrecidos, la construcción de un instrumento que los evalúe a todos suele ser una tarea bastante difícil. Teóricamente, un instrumento puede cumplir con todos los requisitos, sin embargo, la realidad muchas veces es más compleja y supera a la capacidad de evaluación del instrumento. Por ejemplo, si pensamos en medir las diferencias entre las bibliotecas de los distintos programas, el solo hecho de evaluar si los títulos corresponden al nivel deseado en cuanto actualidad, relevancia $u$ otras variables, podría ser una tarea que nos llevaría mucho tiempo.

Por otra parte, efectivamente los rankings no son instrumentos científicos, es decir, no buscan probar o rechazar una hipótesis; sólo buscan informar y evaluar el estado del mercado de MBA en alguna de sus versiones. No existe ningún ranking que haya evaluado las distintas versiones de programas de MBA dictadas por cada escuela de negocios, ya que requiere un esfuerzo en tiempo y dinero que ninguna institución está dispuesta a asumir. Además, que no exista un listado exhaustivo y público de alumnos, graduados y actores relevantes en el mercado de MBA, implica que la aleatoriedad de las muestras sólo cumple con normas ficticias, ya que efectivamente las muestras pueden ser aleatorias, pero como la calidad de la información censal no está asegurada no se pueden deducir ni proyectar los resultados obtenidos: sólo se limitan a expresar lo que los entrevistados señalaron (Moser y Kalton, 1971, pp. 1-187).

Sin embargo, a pesar de estas limitantes, los rankings sí cumplen con otras dos normas relevantes de cualquier investigación social ${ }^{3}$ :

3 Más información sobre los requisitos para que una investigación pueda ser considerada como científica en: King, Gary; Keohane, Robert y Verba, Sidney, Designing social inquiry: scientific inference in qualitative research, Princeton University Press, Princeton, 1994, cap. 1 y 2; Maxim, Paul. Quantitative Research Methods in the Social Sciences, Oxford University Press, New York, 1990, cap. 1 y 2; y Lieberson, Stanley, Making it Count: The Improvement of Social Research and Theory, University of California Press, Berkeley, 1985. 
validez y confiabilidad de las medidas, y estrategia de recolección de datos conocida.

La validez y confiabilidad de los datos se dan en la medida en que el diseño muestral, el instrumento a ser aplicado y el tipo de análisis a ser realizado puedan ser replicables y comunicados. Cualquier estudio de este tipo debe tener sus metodologías abiertas al conocimiento público, en eso se basa gran parte de su confiabilidad. Otra parte de ésta se basa en la consistencia en los resultados, es decir, que cualquiera pueda replicar el mismo instrumento, entrevistar a las mismas personas y obtener básicamente el mismo resultado. Los rankings más conocidos explican, paso a paso, tanto las variables a ser evaluadas como la forma en que éstas serán medidas y, posteriormente, qué método utilizarán para la realización del ranking (análisis de datos).

Adicionalmente, otro elemento que le entrega confiabilidad a los datos recolectados es que la fuente de información sea públicamente conocida. En este caso, cuando los rankings incluyen información de los programas MBA, como número de profesores, horas lectivas, investigaciones, entre otros, ésta es provista por las mismas escuelas y posteriormente son fiscalizadas de manera de comprobar la veracidad de los datos ${ }^{4}$.

A pesar de esto, como ya señalamos, no todos los rankings miden lo mismo, algunos le dan más importancia a la opinión de los reclutadores, otros a los de los alumnos, otros a la evidencia mostrada por cada programa; lo cual significa que, mirados desde una perspectiva u otra, los programas se encontrarán en mejores o peores posiciones dentro del ranking. Ello ha generado la resistencia de parte de muchas escuelas por participar en estas mediciones ${ }^{5}$. Por ejemplo, si miramos el ranking de Wall Street Journal (WSJ) vemos que no evalúa al staff de profesores, como sí lo hacen los rankings de The Economist y Finantial

4 Para el mercado local, tanto el ranking realizado por MV Amrop y publicado por revista Capital, como el ranking realizado por la revista América Economía, realizan una visita a terreno donde se comprueba que la información entregada en los cuestionarios sea verídica. Ambos rankings excluyen cualquier información que no cuente con suficientes pruebas para ser validado.

5 "Escuelas cuestionan metodologías usadas en rankings de MBA" en El Diario Financiero, 1 de octubre 2004. 
Times (FT). Adicionalmente, si bien estos últimos miden esta variable a partir de información de las escuelas, a su vez le entregan distinta ponderación. En el caso de FT el staff de profesores tiene un 5\% del total de la evaluación y en The Economist un 8.33\% dentro de una dimensión mayor: la calidad de profesores, la cual tiene una ponderación de $25 \%$ dentro de otra mayor: desarrollo personal y experiencia educacional, la cual tiene, finalmente, una ponderación de $35 \%$ en el ranking total.

Lo importante en este tipo de ranking es mirar los resultados a partir de la variable que adquiere mayor ponderación, porque ésta es la que está señalando qué es lo que el ranking está evaluando. En el caso de WSJ, el ranking se basa en la opinión de los reclutadores, lo que nos indica que no es uno específico de calidad, sino que mide la variable prestigio a través de las experiencias que los reclutadores han tenido con los alumnos de diversas escuelas. Por otra parte, FT le entrega una ponderación muy importante al salario que ganan los exalumnos, por lo tanto tampoco evalúa la calidad del programa en sí, sino que lo hace desde el punto de vista del mercado, es decir, si sus alumnos vienen de las escuelas más prestigiosas se espera que estén en los mejores puestos de trabajo y que el cambio en su salario sea muy significativo. Por lo tanto se asemeja mucho más a un ranking de prestigio de las marcas que a un ranking de calidad. El ranking The Economist es el que más se asemeja a uno de calidad de programas, ya que mide variables más heterogéneas, dándole preponderancia al desarrollo de carrera posterior y a la experiencia personal y educacional.

Por esta razón es que, al leer un ranking, siempre hay que mirar el contexto en el que los datos están siendo dados a conocer, ya que sacarlo del mismo puede implicar informar erróneamente a la opinión pública.

Para tener una visión de la heterogeneidad de las mediciones existentes, el cuadro a continuación muestra el diseño, las variables medidas y las ponderaciones entregadas a cada ítem de algunos de los estudios internacionales más conocidos. 


\section{Cuadro 1.}

\section{COMPARACIÓN METODOLOGÍAS DE RANKINGS INTERNACIONALES MBA ${ }^{6}$}

\section{Rankings internacionales}

Finantial Times (FT)

Dimensiones evaluadas:

- Performance del programa MBA en cuanto a salario, avance en la carrera profesional del egresado y efectividad de los servicios de la bolsa de trabajo de cada escuela.

- Diversidad: en cuanto a profesores, directores /autoridades, cantidad de alumnos que lograron trabajo fuera del país de origen de la escuela y el suyo propio, experiencia internacional ganada por viajes, intercambios, entre otros, porcentaje de extranjeros y mujeres en los directorios de las escuelas y entre los estudiantes.

- Performance: evaluada en cuanto a publicaciones en 35 journals internacionales después de 1999.

Método de recolección de información:

- Entrevista a egresados de MBA hace 3 años.

- Entrevista a las escuelas.

Ponderaciones: Cada criterio es evaluado con puntajes Z (Z-scores) lo cual entrega no sólo las diferencias entre cada escuela de negocios sino que también la dispersión de los puntajes.

- Salario ponderado (20\%): Promedio salario actual ajustado según la variación entre sectores de la economía.

- Incremento porcentual del salario (20\%)

- Retorno de la inversión (3\%)

- Progreso en la carrera (3\%)

- Logros alcanzados (3\%)

- Éxito en emplearse (2\%)

- Empleado a 3 meses de egresado (2\%)

- Mujeres en el staff (2\%)

- Estudiantes mujeres (2\%)

- Mujeres en el board (1\%)

- Extranjeros en el staff (4\%)

- Extranjeros en el board (2\%)

- Movilidad internacional (6\%)

- Experiencia internacional (2\%)

- Idiomas requeridos (2\%)

- Staff con PhD (5\%)

- Graduados con PhD dentro de los últimos 3 años (5\%)

- Publicaciones (10\%)

6 En el presente cuadro sólo se muestran algunos rankings internacionales a modo de ejemplo. Los rankings internacionales evalúan las escuelas como un todo, tomando en consideración los alumnos de programas full-time. 
Continuación Cuadro 1

\section{Wall Street Journal (WSJ)}

Dimensiones evaluadas: 27 atributos en los que se fijan los reclutadores.

Método de recolección de información: Entrevistas a 2.221 reclutadores.

Ponderaciones: Cada escuela necesitó un mínimo de 20 respuestas para entrar al ranking. El puntaje final usó una combinación de resultados del año actual y el año anterior.

- 75\% corresponde a las evaluaciones de 12 atributos de las escuelas y 12 de los estudiantes.

- 5\% corresponde a la percepción de la escuela y estudiantes como un todo.

- 20\% al número de reclutadores que reclutan directamente desde las escuelas.

\section{The Economist}

Dimensiones evaluadas:

- Oportunidades nuevas en el desarrollo de carrera (35\%):

- Diversidad de reclutadores.

- Éxito en emplearse dentro de 3 meses.

- Trabajo encontrado a través de la bolsa de trabajo.

- Evaluación de estudiantes de la bolsa de trabajo.

- Desarrollo personal y experiencia educacional (35\%)

- Calidad del staff: proporción de profesores v/s estudiantes, \% profesores $\mathrm{PhD}$, evaluación de estudiantes del staff.

- Calidad de estudiantes: promedio GMAT, promedio años de experiencia laboral.

- Diversidad de estudiantes: \% de estudiantes extranjeros, \% de estudiantes mujeres, evaluación de estudiantes del ambiente cultural y compañeros.

- Experiencia educacional: evaluación de estudiantes del contenido y cursos electivos, rango de programas de intercambio al extranjero, cantidad de idiomas ofrecidos, evaluación de la infraestructura y servicios.

- Aumento en el salario (20\%):

- Aumento del salario después de graduados.

- Salario a la salida del MBA.

- Red de contactos (10\%):

- Fuerza de la red de graduados: proporción de alumno v/s alumnos actuales.

- Internacionalidad de la red: proporción de alumnos extranjeros egresados.

- Efectividad de la red: evaluación de alumnos de la red de egresados.

\section{Método de recolección de información:}

- Entrevista a escuelas.

- Entrevista a 23.285 alumnos actuales y recién graduados.

Ponderaciones: las evaluaciones de los estudiantes y egresados pesan un $20 \%$ del total del ranking, y $80 \%$ se basa en la información que las escuelas entregan. 
Como hemos señalado, estos rankings generan sentimientos encontrados: los defienden aquellos que son bien evaluados, pero basta que alguno no los evalúe como ellos esperan para que los critiquen y hasta retiren su participación. Este año 2004 algunas escuelas, como Harvard y Wharton rechazaron participar en cualquier ranking, lo cual afecta a estas mediciones por cuanto son las propias escuelas las que entregan la información acerca de sus programas y facilitan las bases de datos de los alumnos y ex alumnos que serán entrevistados. Como señala The Economist ${ }^{7}$, esto afecta principalmente a aquellos rankings cuya principal fuente de información son los alumnos. En su caso en particular, para incluir a los alumnos de aquellas escuelas que decidieron no entregar información, diseñaron una base de datos basada en las respuestas pasadas e incluyeron las opiniones de los alumnos que aún lograban cumplir con el criterio de haber egresado en los últimos tres años, entregándole una mayor ponderación a la generación más reciente. Esto nos muestra que, a pesar de que las escuelas no quieran ser medidas, el mercado se las ingeniará para evaluarlas ya que es una demanda que los consumidores necesitan satisfacer.

\section{ACREDITACIONES INTERNACIONALES}

Con anterioridad a los rankings, y como contrapartida a la información que éstos publican, nacen, desde la misma industria de los MBA, las asociaciones que certifican estos programas en el contexto mundial. Dado el carácter internacional de estos programas, principalmente aquellos dictados en Estados Unidos y el Reino Unido, es que la certificación y acreditación también están bajo estándares internacionales. Estas asociaciones, dentro de las cuales las más conocidas son $\mathrm{AACSB}^{8}, \mathrm{AMBA}^{9}$ y EFMD ${ }^{10}$, certifican que los programas cumplan con los requisitos mínimos de calidad, por lo cual se convierten en un instrumento que permite medir con la misma vara programas que tienen distinto formato, por ejemplo executive y fulltime, como también aquellos que se dan en contextos locales muy disímiles.

7 [Sitio en Internet] Disponible en http://mba.eiu.com/site_info.asp?info_name=wmba_rank_method

8 The Association to Advance Collegiate School of Business. www.aacsb.edu.

9 The Association for MBAs.

10 European Foundation for Management Development. 
Una acreditación internacional asegura que la escuela dicta un programa que es reconocido en distintos lugares del planeta. Sin embargo, y a pesar del rigor que estas acreditaciones exigen (ya que las acreditaciones se hacen programa a programa lo que significa que no es la escuela de negocios la acreditada sino que cada uno de los programas ofrecidos por ella) y de que nos permiten contar con información detallada para cada uno de ellos -lo cual no permite los rankings-, también poseen una fuente de asimetría importante que es el costo. Éste tiene gran implicancia en un mercado como el nuestro, ya que sólo algunos actores logran acceder a ella. Una consecuencia no menor es que, al no existir una acreditación local validada por los mismos actores del mercado nacional, la asimetría de información es aún mayor dejando a los rankings gran parte de una responsabilidad que debiera ser compartida por distintas instituciones.

\section{LA SITUACIÓN DEL MERCADO CHILENO}

En Chile, el tema de la asimetría de la información, en cuanto a lo que cada programa MBA ofrece, también se ha posicionado en el mercado educacional de posgrados. ¿Qué significa acreditación internacional? ¿Es lo mismo un convenio de doble titulación y que el programa en sí sea una doble titulación? Estas son dudas que pueden surgir a la hora de elegir un programa. Ante éstas, y debido a la falta de información que caracterice la oferta específica de programas MBA dirigida hacia los consumidores, en Chile los rankings han comenzado también a proliferar. 
Cuadro 2

Comparación Metodologías Rankings MBA Regionales y Nacionales ${ }^{11}$

\section{Rankings regionales}

\section{América y Economía}

Dimensiones evaluadas:

- Excelencia en la selección de candidatos: puntaje GMAT promedio, nivel de selectividad (\% de candidatos aceptados en programa).

- Relación de la escuela con América Latina: expresado en esfuerzos por fortalecer y ampliar vínculos con la región.

- Innovación curricular: medida a través de la incorporación de nuevos contenidos, estructura del programa de estudios y metodologías de enseñanza.

- Internacionalización: nivel de participación de alumnos extranjeros en el programa full-time.

Método de recolección información:

- Entrevista a 302 decanos y profesores de escuelas de negocios de América Latina, EE.UU., Canadá y Europa (Medición Prestigio).

- Entrevista a 75 instituciones que se consideraron interesantes ya sea por su calidad general y/o su foco global.

Ponderaciones: todas las variables tienen igual peso.

2. Rankings nacionales

MV Amrop- Revista Capital

Dimensiones evaluadas:

- Infraestructura: cantidad de salas de clases y estudio (10\%), calidad infraestructura salas de clases (10\%), equipamiento tecnológico salas de clase y estudio ( $10 \%)$, entrega de material y bibliografía (20\%), percepción alumnos sobre infraestructura (30\%).

- Redes Internacionales: acreditación internacional (25\%), convenios de doble titulación (12.5\%), Proporción de alumnos que realizaron convenios de doble titulación en el último año (12.5\%), alumnos que estudiaron en el extranjero por convenios con universidades extranjeras durante el último

11 En el ámbito regional sólo conocemos el ranking que realiza la revista América- Economía; en el nacional existe el ranking realizado por Qué Pasa, el cual mide la satisfacción de los ex alumnos con los programas realizados. Este programa no fue incluido en el cuadro por no contar con información detallada de su metodología. 
año (10\%), alumnos extranjeros recibidos durante el último año (10\%), percepción de alumnos de relaciones internacionales MBA cursado (30\%).

- Redes de Contacto: existencia asociación de ex alumnos (30\%), tipo de actividades realizadas (20\%), frecuencia de actividades realizadas (10\%), convenios con organizaciones en Chile (10\%), percepción de alumnos de la red de contactos MBA cursado (30\%).

- Calidad de Alumnos: requisitos de Admisión (20\%), años de experiencia laboral alumnos (10\%), alumnos con cargo ejecutivo (30\%), cantidad de postulantes (10\%), evaluación alumnos de sus compañeros (30\%).

- Calidad Docente: No horas lectivas (5\%), profesores PhD (10\%), profesores Magíster (10\%), proporción de alumnos v/s profesores (5\%), profesores visitantes (5\%), profesores chilenos de visita en universidades extranjeras (5\%), profesores consultores y/o directivos de empresas $(5 \%), \mathrm{N}^{\circ}$ consultorías e investigaciones (10\%), publicaciones en journals (5\%), publicaciones ISI (10\%), percepción de alumnos de la calidad de sus docentes (30\%).

- Prestigio de Marca: evaluación alumnos y ejecutivos mejor programa dictado en Chile (25\%), recomendación alumnos de su programa MBA (25\%), prestigio MBA en ejecutivos (25\%), preferencia postulación alumnos MBA (25\%).

Método de recolección de información:

- Encuesta a programas MBA

- Encuesta opinión alumnos de los programas participantes (478)

- Encuesta opinión ejecutivos (120)

\section{Ponderaciones:}

- Infraestructura (10\%)

- Redes Internacionales (10\%)

- Redes de Contacto (10\%)

- Calidad Docente (23.3\%)

- Calidad Alumnos (23.3\%)

- Prestigio de Marca (23.3\%)

En el ámbito nacional nos encontramos con dos tipos de mediciones: regionales y nacionales. Las primeras, si bien nos evalúan en perspectiva comparada con el resto de América Latina, se han caracterizado por tener principalmente dos problemas: por una parte comparan programas de distinto formato ${ }^{12}$, señalando que la medición se hace considerando escuelas de negocios y, por otra, no son incluidas (o invitadas) en las mediciones todas las existentes. Esto significa que, localmente, sólo es posible conocer la realidad de algunos programas; en cierta medida son los más prestigiosos, pero que no dejan cabida

12 En estas mediciones se comparan programas full-time y part-time, ya que no todas las escuelas elegidas dictan programas full time. 
para mostrar el dinamismo del mercado y el posicionamiento de nuevos programas en el ámbito local.

En el caso de los rankings nacionales, éstos aún se centran en medir la calidad del programa bajo diferentes perspectivas. A pesar de que esto podría derivar en que tendieran a medir las mismas variables, ello no es así, y tal como vimos en el caso de los internacionales ya comienza a producirse una diferenciación importante entre ellos. De esta forma observamos que el ranking MV AMROP, que publica revista Capital, se basa en la información de las escuelas, en la evaluación que realizan los alumnos y en la evaluación que realiza el mercado a través de los ejecutivos; en cambio el publicado por revista Qué Pasa es un ranking que sólo mide la satisfacción de los ex alumnos de programas MBA.

Como el costo de realizar un programa full-time es muy alto, no sólo en dinero sino también en tiempo -ya que significa dejar de trabajar y darle dedicación exclusiva al MBA-, los formatos de programas executive y part-time han tenido gran éxito en el mercado local, lo cual implica que existen cierto tipo de mediciones que para nuestro mercado no serían relevantes de realizar. Si tomamos el caso de FT donde la mayor ponderación se da en el cambio de salario, para Chile esto sería infructuoso de medir, ya que el mercado nacional aún no le entrega la valoración que sí tienen en el extranjero, principalmente en Estados Unidos y Europa. Otro ejemplo es lo que pasaría si quisiéramos evaluar a través de los reclutadores: como la mayoría de las personas que estudian programas MBA está trabajando, el tema de las bolsas de trabajo no adquiere la misma ponderación, por lo tanto la opinión de estos actores pasa a tener menor peso en el ámbito nacional que el que tiene en los mercados de los que podríamos llamar "países desarrollados".

Sin embargo, hay un elemento que, en el caso nacional, adquiere importancia: la red de contactos. En definitiva, los alumnos nacionales, para poder buscar mejores expectativas de trabajo -y carrera en general-, necesitan una red que les permita acceder no sólo a las personas adecuadas, sino que a los negocios adecuados. Ese es, en definitiva, el elemento por el cual estos programas adquieren valor para el mercado. 
Uno de los elementos más difíciles de fiscalizar en el mercado local es, quizás, la tasa real de rechazo de los estudiantes, ya que, como aún el mercado no está maduro, el mayor prestigio está concentrado, básicamente, en dos instituciones que son, a su vez, aquellas que reciben mayor número de postulantes y, por lo tanto, las que concentran una tasa de rechazo más alta. Este fenómeno impide medir la tasa real en aquellos programas que aún están en etapa de formación. Otro tema importante de mencionar, en esta asimetría de prácticas, son las evaluaciones que los programas realizan para seleccionar a sus alumnos. La variedad de pruebas es inmensa: de conocimientos relevantes, habilidades, rasgos psicológicos e idioma, entre otras, por lo que los niveles de complejidad de éstas son igualmente variados. Esto significa que, a diferencia de lo que pasa en los programas internacionales, no existe una medida homogénea (por ejemplo, puntaje GMAT (Graduate Management Admission Test) para evaluar la calidad de los alumnos), debiendo crear estandarizaciones para medir las diferencias reales entre las distintas pruebas, e/o incluir otras variables relevantes observadas en el mercado local para poder obtener una evaluación que se aproxime más fielmente a la realidad.

Como parte de la reacción que ya se ha observado internacionalmente, en Chile existen también algunos programas que no participan de estas mediciones y otros que se retractan en su participación. Esto, como vimos, no llevará a que se terminen los rankings -ni en Chile, ni en la región, ni en el resto del mundo-, sino a que los programas comiencen a ser medidos desde otras fuentes de información que no dependan directamente de las escuelas. Ello llevará a que las variables medidas serán mucho más subjetivas, ya que, actualmente, al existir la colaboración de las escuelas, al menos éstas se aseguran que parte importante de la información no dependa de las opiniones y/o percepciones de terceros sino en información objetiva y verificada.

En cuanto a las acreditaciones como ente fiscalizador de la oferta, parece importante que exista alguna instancia, no necesariamente estatal, en la cual todos los actores involucrados pudieran ponerse de acuerdo sobre cuál es el mínimo que deben cumplir aquellas instituciones que deseen dictar programas MBA; de esta manera, no serán sólo los rankings o encuestas de opinión las que nos entregarán una visión del mercado local, sino que se contará con información debidamente certificada por los mismos actores del mercado. 
Actualmente, si bien Comisión Nacional de Acreditación de Postgrado (CONAP) cumple este rol, la mayoría de los programas MBA no han realizado este proceso por diversas razones: en primer lugar, algunos señalan que las condiciones de CONAP son restrictivas, ya que sólo se aplican a universidades autónomas; en segundo lugar, esta Comisión tiene una normativa orientada a la evaluación de programas con un contenido altamente académico, lo cual implica que muchos programas de MBA, dada su naturaleza, no cumplirían con los requisitos básicos señalados por ella ${ }^{13}$. Esto genera otra fuente adicional en la asimetría de información, en que los programas acreditados defienden el haber podido cumplir con esta normativa; sin embargo, los no acreditados señalan que el sólo hecho de ser universidades autónomas les confiere la facultad de dictar programas de posgrado, cualquiera sea su naturaleza.

En este sentido, el mercado chileno aún está a tiempo de lograr un buen equilibrio entre información pública realizada por entes privados y una institución fiscalizadora, la cual pueda quizás surgir desde las mismas escuelas de negocios. El funcionamiento de ambas (ranking y acreditación) ayudará a que este mercado se regule y funcione con menores niveles de desconfianza que los actualmente observados, y con mejores niveles de información al consumidor.

13 Los programas actualmente acreditados en CONAP son Magíster en Administración de Empresas, de la Pontificia Universidad Católica de Chile, y Magíster en Gestión y Dirección de Empresas, del Departamento de Ingeniería Industrial de la Universidad de Chile. 


\section{REFERENCIAS BiBLIOGRÁFICAS}

Moser, Claus y Kalton, Graham, Survey Methods in Social Investigation, Heinemann Educational, London, 1971.

King, Gary; Keohane, Robert y Verba, Sidney, Designing social inquiry: scientific inference in qualitative research, Princeton University Press, Princeton, 1994.

Maxim, Paul, Quantitative Research Methods in the Social Sciences, Oxford University Press, New York, 1990.

Lieberson, Stanley, Making it Count: The Improvement of Social Research and Theory, University of California Press, Berkeley, 1985.

"Escuelas cuestionan metodologías usadas en rankings de MBA", en El Diario Financiero, 1 de octubre 2004.

MV Amrop, "Informe Ejecutivo. Benchmarking MBA MV Amrop 2004", en: revista Capital, No 137, Santiago, 2004. 